\title{
Seashore GPS Positioning Intelligent Monitoring System
}

\author{
Hu Yingkun, Zhong Yuling, Guan Taian \\ Guangdong Polytechnic of Industry \& Commerce, Guangzhou, China \\ Email address: \\ 523792534@qq.com (Hu Yingkun)
}

\section{To cite this article:}

Hu Yingkun, Zhong Yuling, Guan Taian. Seashore GPS Positioning Intelligent Monitoring System. International Journal of Sensors and Sensor Networks. Vol. 7, No. 2, 2019, pp. 23-27. doi: 10.11648/j.ijssn.20190702.12

Received: August 6, 2019; Accepted: August 28, 2019; Published: September 12, 2019

\begin{abstract}
In some large scenic spots, such as seashore tourists, there may be accidents such as loss. In addition, in order to facilitate the management and monitoring of tourists, the project designed a system for seashore tourists positioning. Firstly, this paper introduces the overall design scheme of the system, in which the positioning tag uses MC13224 chip as the main processor, transmits GPS information to the positioning gateway through ZigBee communication mode, and receives information from the server side. Then, the hardware design block diagram and software design flow of the gateway and the base station are analyzed respectively. The validation shows that the location tag can send the location information to the server and receive the information from the server. It realizes that tourists can receive the information from the server. The client can monitor the location of tourists and alarm information. Finally, the application value of the system is analyzed.
\end{abstract}

Keywords: GPS Positioning, LABEL, Gateway, Base Station

\section{Introduction}

In some large scenic spots, such as seashore tourists, sometimes tourists lose their places, and the situation of lost and found tourists appears. In addition, the managers of scenic spots need to inform the tourists of some news, such as weather forecast, itinerary arrangement and so on. In the same type of products at home and abroad, GPS data is sent through mobile network or satellite network, which costs a lot. Based on this, this project designs a seashore tourist positioning system. Data transmission is a local area network composed of ZIGBEE, which has low cost and great competitive advantage. Tourists wear electronic tags on their wrists (hereinafter referred to as tags), which resemble a watch. Through the GPS positioning module, the positioning information is sent to the monitoring center through the local area network composed of ZigBee [1]. When they encounter an emergency, they can send an alarm to the monitoring center through the alarm button, and receive the broadcast information sent by the monitoring center, such as lost and found, weather information, route arrangement, etc.

When the positioning label works normally, it should make the positioning label join the network successfully in the areas that need to be monitored, and positioning can be successful. The display screen can display weather forecast, alarm and other information. The server can send messages to the positioning label, and can monitor the mobile location of all the positioning labels.

\section{General Scheme of System Design}

The overall scheme of the system is shown in Figure 1. The structure and functions of the whole system are as follows: 1. Location gateway is responsible for networking, collecting positioning data and reporting positioning data to positioning server; 2 . Location base station is responsible for forwarding positioning data of positioning terminal; 3 . Location terminal is responsible for real-time reporting information needed for positioning, displaying time and displaying positioning data under monitoring center. The monitoring center is responsible for label and base station configuration information management.

The advantages of this scheme are as follows: 1. Because ZIGBEE protocol stack is not used [2], the procedure flow of the positioning terminal is more concise, and the application layer can flexibly control the dormancy of the positioning terminal, which will make the positioning terminal more power-saving; 2. To realize the networking process through MAC, the positioning terminal can quickly cut between different base stations. In order to avoid the phenomenon of label dropping off for a long time when switching base station [3]. 


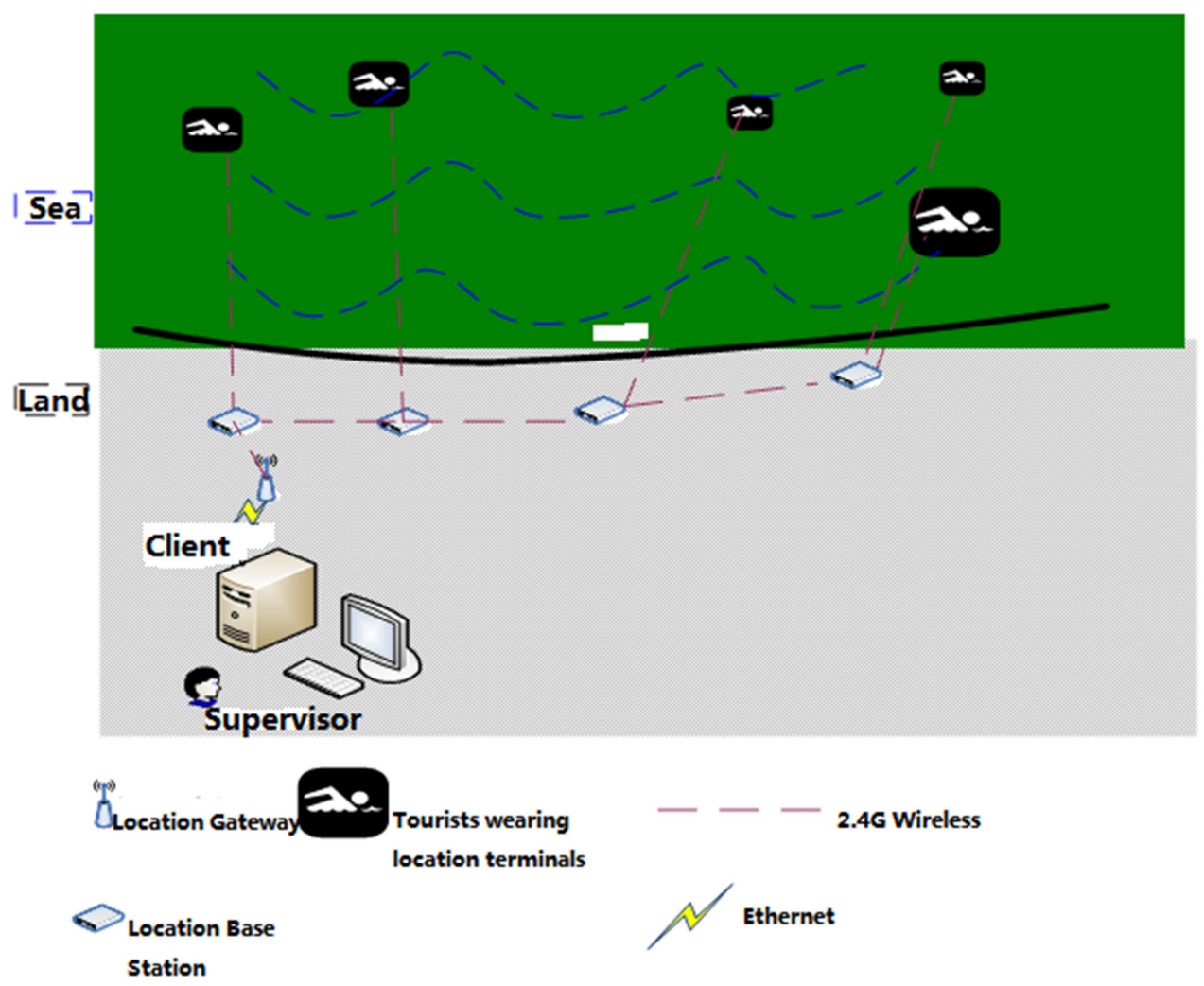

Figure 1. System Overall Scheme.

\section{Hardware and Software Design of System Composition}

\subsection{Principle Block Diagram and Software Design of Positioning Terminal}

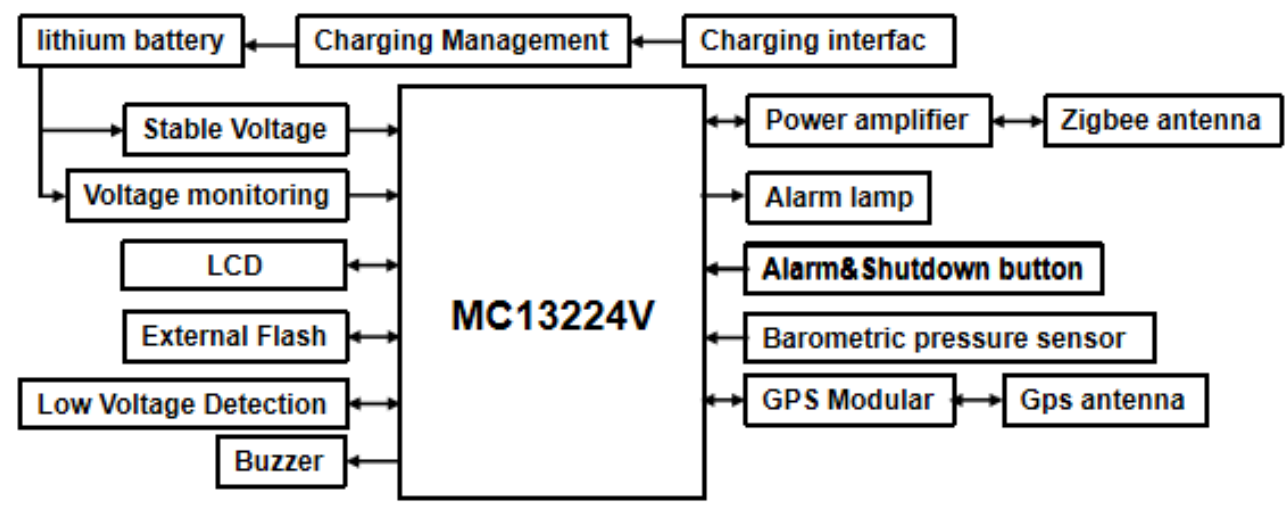

Figure 2. Principle block diagram of positioning terminal.

The principle block diagram of the positioning terminal is shown in Figure 2. Because the location terminal is worn in the hands of tourists, the demand for low power consumption is very high, so the selection of battery is very important, considering the size, price, capacity, calorific value of the battery, etc. [4]. The selection of batteries in this system is as follows:

The transmitting current of the positioning terminal is 170 $\mathrm{mA}$, the transmitting time is $10 \mathrm{~ms}$, and the data is sent once a second. The static working current of the positioning terminal is $10 \mathrm{~mA}$, and the working current of the GPS module is $35 \mathrm{~mA}$ [5]. Mean current:

$$
\begin{gathered}
\overline{\mathrm{I}}=\frac{\text { Electric quantity }}{\text { Use time }}=170 \times 10 \times 10^{\wedge}(-3)+35+10 \\
=46.7 \mathrm{~mA}
\end{gathered}
$$

16-hour power consumption: (calculated at 90\% usage)

$$
\overline{\mathrm{I}}=\frac{\text { average current }(\mathrm{mA})}{\text { Use time }(\mathrm{h})}=46.7 * 16 / 0.9=830 \mathrm{mAH}
$$

The selected 474147-900mAh, lithium battery, with charge 
and discharge protection board, capacity $900 \mathrm{mah}$, meets the requirements [6].

The main chip is a MC13224 chip developed by Freescale [7]. This ZigBee chip integrates a complete low-power 2.4 $\mathrm{GHz}$ radio transceiver, 32-bit ARM7 processor, a hardware accelerator for secure encryption of 802.15.4, MAC and AES, and a complete set of MCU peripherals. It is a high-density and low-component number of IEEE 80. 2.15.4 integrated solution, in order to expand the coverage of the signal, an additional power amplifier chip [8]; GPS positioning chip uses A2200, positioning accuracy is very high. LCD uses LM128128CBY monochrome screen [9], with a backlight current of $40 \mathrm{~mA}$ and no backlight current of less than $1 \mathrm{~mA}$. The text on the screen can also be clearly seen without backlighting when there is external light. Because the location terminal should be able to display Chinese characters, the internal flash of MC13224 only has 96K, and the application occupies about $90 \mathrm{~K}$, basically no extra space. So we need to expand flash. The size of Chinese fonts and interface pictures is about $400 \mathrm{~K}$ bytes. Extended flash chip W25Q16CVSSIG has 2M bytes of space, which is connected with MC13224 by SPI interface [10]; buzzer is used to receive server information dripping, reminding tourists of the need to view messages; two buttons are used to turn on and off the screen; one is used for emergency alarm.
The software design flow of the positioning terminal is shown in Figure 3. First, the main chip MC13224 initializes, configures the initialization parameters, and actively looks for the local area network formed by the gateway. After successfully joining the network, the timer is turned on for $1 \mathrm{~s}$ [11], and the LCD display is turned on. The displayed information includes weather forecast, time, current residual electricity and charging indication, whether there is connection or not. Receive the information sent by the monitoring center; after initialization, use the highest priority external interrupt to judge whether to press the shutdown button, press long or short, press long to execute standby commands, press short to turn on the power supply of each module, that is, open the screen; and then use the secondary priority external interrupt as an alarm button, such as If an alarm interruption occurs, the command to send an alarm frame to ZigBee is executed and the LED flicker is turned on at the same time; the battery voltage is detected every 6 seconds, and if the battery power is less than $10 \%$ of the total power, the battery icon flickers to remind the user to charge; if ZigBee receives the data from the base station, the corresponding content is displayed on the LCD and concurrently displayed. Send reply frames, or wait for data to arrive; if 1 second time has arrived, then get GPS positioning data and send it to base station [12] through ZigBee.

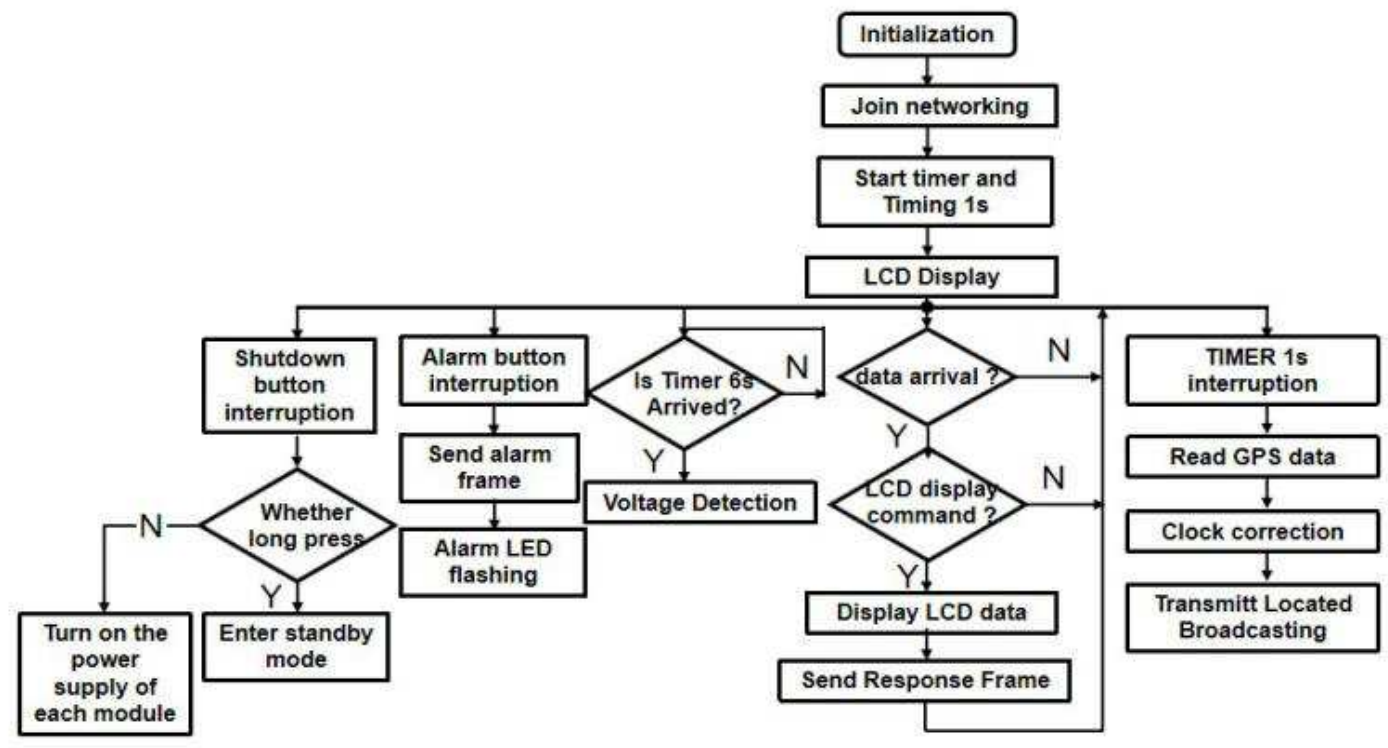

Figure 3. Software design process of positioning terminal.

\subsection{Principle Block Diagram and Software Design of Gateway}

Choose the IPORT module developed by Zhou Ligong Company. The technology of this module is very mature. It can quickly and steadily realize the function of serial port to Ethernet, meet the needs of the project, and has its own configuration tools. Its main functions are as follows: it is responsible for forming a local area network for each base station, using ZigBee wireless communication mode to communicate with each base station, and then forwarding the data to the monitoring center, or receiving the data from the monitoring center, playing the role of a repeater [13]. Its hardware and software design are shown in Figures 4 and 5.

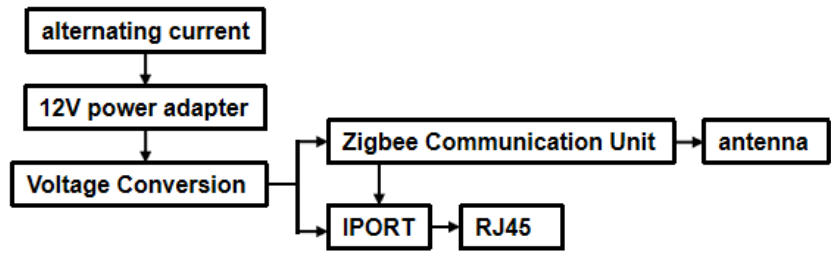

Figure 4. Principle block diagram of gateway. 


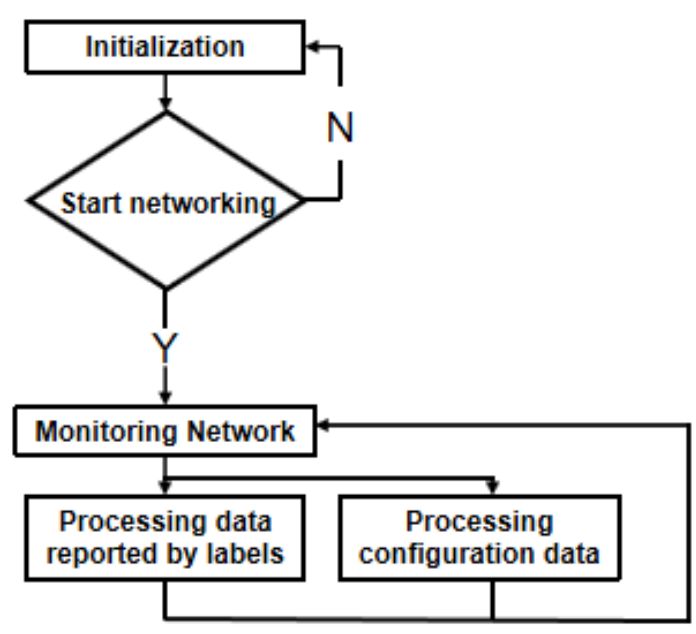

Figure 5. Gateway Software Design.

\subsection{Principle Block Diagram and Software Design of Base Station}

MC-Z03-3001 ZigBee communication unit module is used. Its main work is to send and receive label information and to communicate with gateway wireless [14]. Its hardware and software design are shown in Figures 6 and 7.

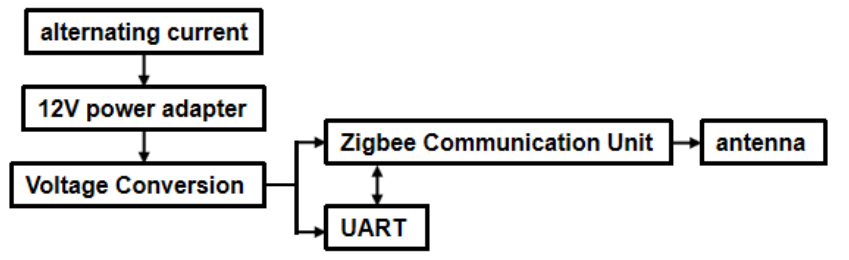

Figure 6. Principle block diagram of base station.

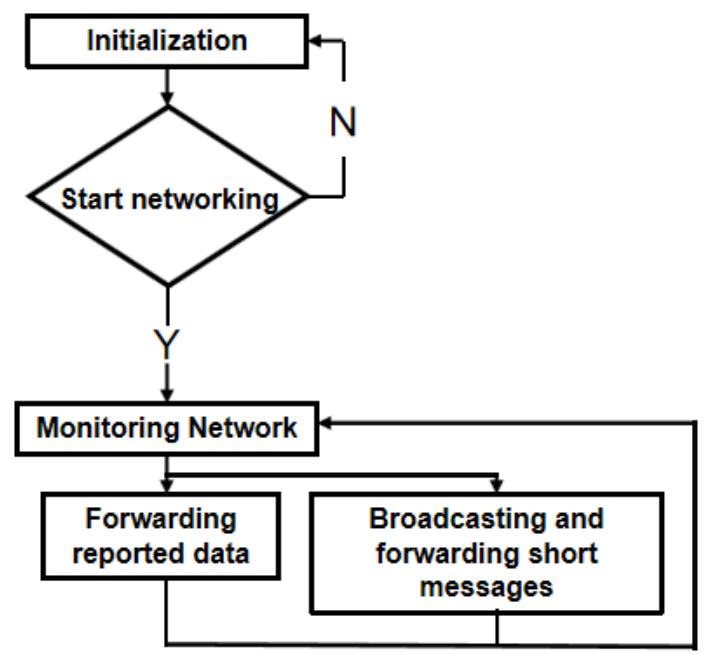

Figure 7. Software Design of Base Station.

\section{System Construction and Use}

\subsection{Principle Block Diagram and Software Design of Positioning Terminal}

1. Parameter Configuration

The system includes PC host computer, location gateway and location base station parameter configuration. Configure the PAND of the location gateway (configure it by sending AT commands through the serial debugging assistant). Note that in the same network, the location gateway and the location base station PANID cannot be duplicated, and the ID between the location base station and the location base station cannot be duplicated. The wireless coverage of a positioning gateway is 300 meters, which needs to be increased by positioning base stations [15]. It should be noted that: 1 . In the same network, the PANID between the location gateway and the location base station, the location base station and the location base station cannot be repeated; 2 . Before installing the location base station, it needs to be configured. The main configuration items are as follows:

(1) PAND with location base station: $\mathrm{ATPN}=14$;

(2) Configuring the PAND of the target location gateway of the base station: ATDP $=13$ (for data forwarding);

The base station receives the heartbeat packet sent by the location gateway every 3 seconds. The LED display mode is always bright, and the heartbeat packet LED 3 flickers. If the base station is disconnected from the location gateway, the LED 2 and the LED 3 flicker alternately.

2. Location terminal connection preparation

(1) The positioning terminal is connected to the computer through USB cable to charge the positioning terminal. At this time, the charge indicator icon $N$ is displayed on the screen status bar. When the charge is completed, the charge indicator icon becomes

(2) When the positioning terminal works normally, the main interface is shown in Figure 8.

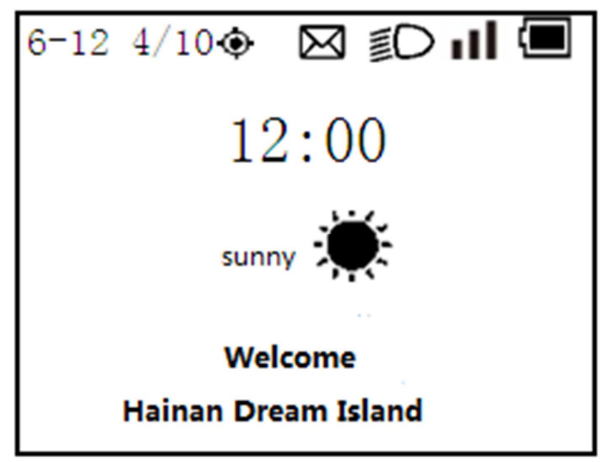

Figure 8. Main interface.

If the signal icon on the screen of the positioning terminal is displayed as follows $\mathbf{I l}$, it indicates that the positioning terminal has successfully entered the network. Appropriately adjust the location of the location gateway and the location base station, so that the location terminal can successfully join the network in the areas that need to be monitored.

\subsection{System Use and Functional Verification}

The test must be carried out when the positioning terminal successfully joins the network.

1. Basic Function Testing of Location Terminal

(1) GPS positioning

Placing the positioning terminal in an open outdoor area, 
within one minute, the GPS icon on the display screen (from Pbeing changed into one indicates that the positioning is successful.

(2) Emergency alarm

At the same time, press the alarm button around the positioning terminal, the buzzer of the positioning terminal sounds, the alarm lamp on the positioning terminal flickers, and the icon shown on the positioning terminal screen is as follows. At the same time, the monitoring center will display the alarm status of the positioning terminal, indicating that the function is normal.

(3) Time display

When the GPS signal of the positioning terminal is marked $\phi$, the positioning terminal can display the current time and date normally (whichever is Beijing time).

(4) Screen Backlight

Short press the front key of the positioning terminal, the screen backlight is bright, and the backlight is automatically extinguished after 10 seconds.

(5) Turn off and turn on

Press the front button for a long time, and the interface shows "Confirm shutdown?" By pressing the front button, the positioning terminal will enter the standby state, at which time all the functions of the positioning terminal will stop. In standby state, press the front button and the positioning terminal will start.

2. Personnel Location Function Test

When the GPS signal of the positioning terminal is marked $\phi$, the positioning terminal is moved around, and the movement track of the positioning terminal can be seen through the client, which indicates that the function is normal.

\section{Conclusion}

Location label as a platform product, can be used in factories, parks, scenic spots and other scenarios in the future, the design of the whole system is basically similar; In addition, the advantages of this system compared with the use of mobile phone positioning: 1 . Mobile phones need to be covered by mobile networks to receive and receive data; 2 . Receiving and dispatching scenic spots by mobile phones On the one hand, it is inconvenient for the management center to send the information to all tourists, and it cannot monitor the location of all tourists in real time. The monitoring center of this system can monitor the location of all tourists in real time. When the tourists come out of the effective monitoring range, they will immediately drip, remind the tourists to go over the effective area, and send the alarm information to the monitoring center. When the tourists wear the label in their hands, the buzzer will drip when they receive the information from the monitoring center. We will check the information immediately without missing the valid information; if there is an emergency, we can use the label to alert the monitoring center. This system saves electricity, operates conveniently and flexibly, costs cheaply, and has strong practical significance.

\section{References}

[1] Li Yuanmao, Liu Guixiong, and Zeng Chenggang. Design of outdoor radioactive source information monitoring system based on GPS [J]. Journal of Electronic Measurement and Instruments, 2016, 33 (6): 1244-1253.

[2] Chen Zhiyuan, Zhu Yecheng, and Zhou Zhuoquan. A smart home control system based on STM32 [J]. Application of electronic technology, 2012, 38 (9): 138-140.

[3] Chen Qi, Han Bing, Qin Weijun, and Huang Fuwei. Design and implementation of Internet of Things gateway system based on Zigbee/GPRS [J]. Foreign electronic measurement technology, 2011, 48 (9): 367-372.

[4] Yang Liu, and Mu Hao-miao. An orchard locomotive anti-collision and anti-dumping early warning system based on ZigBee wireless transmission $[\mathrm{J}]$. Journal of Agricultural Engineering, 2014, 30 (21): 25-31.

[5] Wu Wenping, and Wang Ruifeng. Application of GPS-ZigBee integrated positioning in modern tram positioning [J]. Urban Rail Transit Research, 2016, 19 (5): 133-136.

[6] Kongxuehua. Design of an intelligent infusion control system [J]. Foreign electronic measurement technology, 2014, 33 (6): 73-77.

[7] Yao Yunping. Design of intelligent infusion device control system based on single chip computer [J]. Journal of Shaanxi University of Science and Technology, 2010, 28 (4): 83-87.

[8] Sun Shuying. Development and Application of a New Generation Embedded Microprocessor STM32F103 [J]. Microcomputer Applications, 2010, 20 (5): 43-47.

[9] $\mathrm{Lu} \mathrm{Li}$, and Zhang Bo. High-speed network data acquisition system based on embedded TCP/IP protocol [J]. Journal of Instruments and Instruments, 2009, 30 (2): 405-409.

[10] Xuhang. Embedded multi-channel high-speed data acquisition system [J]. Automation and instrumentation, 2013 (1): 148-150.

[11] Chen Hong et al. Development of multi-channel weak voltage signal synchronous acquisition system [J]. Application of electronic technology, 2014 (40): 79-84.

[12] Zhong Wentao. Field Information Detection and Recording System Based on Single Chip Microcomputer [J]. Electromechanical Engineering Technology, 2013, 42 (5): 26-30.

[13] Ding Fan, and Zhou Yongming. Design of wireless multi-channel temperature data acquisition system based on ZigBee [J]. Instrument technology and sensors, 2013 (8): 72-78.

[14] Pan Lingjiao, Zhang Zijia, and Fan Yanhu, etc. Design of 16-channel embedded synchronous data acquisition system [J]. Instrument technology and sensors, 2013 (11): 91-94.

[15] Chen Tao, Guo Deke, Luo Xueshan, and Chen Honghui. A wireless sensor network data collection method based on mobile base station [J]. Journal of National Defense University of Science and Technology, 2011, 33 (2): 49-53. 